\title{
Applicability of the mutation-selection balance model to population ge- netics of heterozygous protein-truncating variants in humans
}

Donate Weghorn ${ }^{1,2, \ddagger *}$, Daniel J. Balick ${ }^{1,2 *}$, Christopher Cassa ${ }^{1,2}$, Jack A. Kosmicki $i^{3,4}$, Mark J. Daly ${ }^{3,4}$, David R. Beier ${ }^{5,6}$, and Shamil R. Sunyaev ${ }^{1,2} \dagger$

\footnotetext{
${ }^{1}$ Brigham and Women's Hospital, Division of Genetics, Harvard Medical School, Boston, MA, USA

${ }^{2}$ Department of Biomedical Informatics, Harvard Medical School, Boston, MA, USA

${ }^{3}$ Analytic and Translational Genetics Unit, Department of Medicine, Massachusetts General Hospital, Harvard Medical School, Boston, MA, USA

${ }^{4}$ Program in Medical and Population Genetics, Broad Institute, Cambridge, MA, USA

${ }^{5}$ Center for Developmental Biology and Regenerative Medicine, Seattle Children's Research Institute, Seattle, WA, USA

${ }^{6}$ Dept. of Pediatrics, University of Washington School of Medicine, Seattle, WA, USA

*These authors contributed equally to this work.

$\ddagger$ Current address: Centre for Genomic Regulation and Universitat Pompeu Fabra, Barcelona, Spain

†Corresponding author: Shamil Sunyaev, ssunyaev@rics.bwh.harvard.edu
}

The fate of alleles in the human population is believed to be highly affected by the stochastic force of genetic drift. Estimation of the strength of natural selection in humans generally necessitates a careful modeling of drift including complex effects of the population history and structure. Protein-truncating variants (PTVs) are expected to evolve under strong purifying selection and to have a relatively high per-gene mutation rate. Thus, it is appealing to model the population genetics of PTVs under a simple deterministic mutation-selection balance, as has been proposed earlier (Cassa et al., 2017). Here, we investigated the limits of this approximation using both computer simulations and data-driven approaches. Our simulations rely on a model of demographic history estimated from 33,370 individual exomes of the Non-Finnish European subset of the ExAC dataset (Lek et al., 2016). Additionally, we compared the African and European subset of the ExAC study and analyzed de novo PTVs. We show that the mutation-selection balance model is applicable to the majority of human genes, but not to genes under the weakest selection.

\section{Introduction}

In well-adapted populations, the evolutionary dynamics of genes under purifying selection plays a prominent role. Early models describing this phenomenon were purely deterministic, that is they assumed infinite effective population size (Fisher, 1930). In particular, the discussion centered 
around the concept of mutation-selection balance, when deleterious variants in the population are replenished by mutation against the constant purge of negative selection. Supported by the advent of large sequencing datasets and computer simulations, however, it became clear that the high amounts of non-silent genetic variation observed in real populations cannot be fully explained by mutation-selection balance (Wright, 1931; Lande, 1976; Bürger et al., 1989). Instead, many mutations are only weakly selected against and many populations cannot be approximated to be infinitely large. Both of these factors emphasize the relative importance of stochastic effects, or genetic drift, compared to mutation and selection. Therefore, deterministic mutation-selection balance is not an adequate description of the evolutionary dynamics of deleterious alleles unless the selection strength is sufficiently high to dominate genetic drift. The full mutation-selectiondrift balance has been extensively studied using the diffusion approximation (Kimura, 1964). It is now widely appreciated that in humans the complexities of demographic history and changing population size must be explicitly modeled. This has been incorporated in many recent studies that estimated the intensity of selection (Williamson et al., 2005; Tennessen et al., 2012; Do et al., 2015). One practically important case when effects of selection are strong in comparison to genetic drift is the dynamics of protein-truncating variants (PTVs). When assuming that most PTVs within a gene have similar fitness effects, they can be analyzed in aggregate. The cumulative number of new truncating mutations per gene is two orders of magnitude higher than the per-site expectation. It is thus appealing to apply a deterministic approximation to model the population genetics of PTVs, as has been proposed by us earlier (Cassa et al., 2017).

The utility and limits of the deterministic approximation are a matter of debate (Charlesworth and Hill, 2019; Cassa et al., 2019). Here, we investigated the applicability of this approximation to selection against heterozygous PTVs, $s_{\text {het }}$, using simulations that explicitly incorporate drift. Further, we studied the effects of population stratification by comparing PTV allele frequencies between the African and non-Finnish European subpopulations of the ExAC dataset. Lastly, we analyzed the estimates of selection strength vis-à-vis the fraction of de novo mutations in a recent pedigree sequencing dataset, a direct measure of $s_{\text {het }}$. Beyond the analysis of the influence of drift on the $s_{\text {het }}$ estimates, we also present a comparison of $\hat{s}_{\text {het }}$ with another measure of protein constraint, pLI (Lek et al., 2016). 


\section{Results}

\section{Impact of genetic drift on PTV allele count}

We first analyzed how genetic drift affects the observed number of PTV mutations on a gene, $k$, and its variance in the population. Given an exome sequencing cohort, $k$ is the sum of proteintruncating mutations over all sampled chromosomes in the cohort, $n$. The expected value of $k, \mathrm{E}[k]$, is determined by selection as well as by the local cumulative mutation rate, $U$. Provided PTVs are not nearly recessive, $\mathrm{E}[k]$ is well approximated by the deterministic expression $n U / s_{\text {het }}$ (Wright, 1931). If mutation and selection are the dominant evolutionary forces, the approximate Poisson nature of the PTV count implies for the variance $\operatorname{Var}[k]=\mathrm{E}[k]=n U / s_{\text {het }}$. Meanwhile, if PTV alleles remain in the population for a substantial period of time and thus become subject to the effects of genetic drift, this will tend to increase $\operatorname{Var}[k]$. We can express the total variance of $k$ as a sum of Poisson sampling variance and variance due to drift (Methods).

The impact of genetic drift shows in the population allele frequency spectrum. For given $U$ and $s_{\text {het }}$, this describes the distribution of the gene-specific cumulative frequency of PTVs in the population, $X$, and depends on the demographic history of the population. To gain intuition, we first considered a classic approximation for the allele frequency spectrum under the assumption of strong purifying selection (Nei, 1968). At equilibrium, the variance of the cumulative PTV allele count $k$ in a sample of size $n$ chromosomes is then given by $\operatorname{Var}[k]=n U / s_{\text {het }}\left(1+n /\left(4 N_{\mathrm{e}} s_{\text {het }}\right)\right)$. Notably, this expression approaches the deterministic approximation of $n U / s_{\text {het }}$ if the size of the sample is much smaller than the product of the effective population size, $N_{\mathrm{e}}$, and the selection coefficient.

\section{Modeling recent population expansion}

Human populations appear to be far from evolutionary equilibrium, and most populations have undergone very rapid, recent growth (Keinan and Clark, 2012). With our focus on rare deleterious PTVs $(\hat{X}<0.001)$, these alleles are expected to be on average young, so the most relevant population size for our purposes is the recent effective population size, corresponding to the lifetime of an average very rare deleterious allele. Current literature estimates of the present day effective size in the European population span the wide range of 0.5 to 8 million individuals (Tennessen et al., 2012; Gao and Keinan, 2014; Browning and Browning, 2015; Harpak et al., 2016). Although the long-term effective size of the human population is smaller than the sample size of the ExAC dataset, this number, driven by ancestral or bottleneck sizes, is less relevant than the epoch of 
recent growth for very young alleles under relatively strong natural selection.

To determine the deviation from the deterministic regime, we evaluated relevant features of the demographic history under which rare deleterious variants evolve. We focused on the Non-Finnish European (NFE) subset, representing a majority of the samples $(\mathrm{N}=33,370)$ in the ExAC data. The NFE subset has a well studied demographic history from a single ancestry. We used features of the Tennessen et al. (2012) model of European demography, including the initial size, bottleneck and initial exponential growth phases. For the final exponential growth phase, we matched properties of rare alleles in the ExAC NFE sample to gauge the final effective population size and corresponding growth rate. We simulated a dense range of exponential growth rates in the final demographic period, and matched neutral simulation results of the downsampled site frequency spectrum to the fraction of synonymous singleton alleles observed in the NFE subpopulation. We focused on non-CpG transversions for these purposes, as $\mathrm{CpG}$ and even non-CpG transitions are known to exhibit the effects of recurrent mutations in the ExAC sample due to elevated mutation rates relative to non- $\mathrm{CpG}$ transversions. For example, the fraction of synonymous singletons for non- $\mathrm{CpG}$ transversions in ExAC NFE is 63.3\% (95\% CI 62.1-64.6\%; ExAC All 60.5\%), while the fraction of synonymous singletons for CpG transitions in ExAC NFE is 39.8\% (39.1-40.7\%; ExAC All 23.9\%). The analysis of singletons resulted in a demographic history with a recent population size of 4.3 million individuals, which is consistent with estimates based on the same dataset provided in Harpak et al. (2016).

In Figure 1(a), we find the coefficient of variation, $\sqrt{\operatorname{Var}[k]} / \mathrm{E}[k]$, to be in good agreement with the deterministic approximation for genes under strong selection (see also Supplementary Figure 1). For $s_{\text {het }}=0.06$, corresponding to the mean of the inferred genome-wide distribution of heterozygous selection coefficients in Cassa et al. (2017), the inflation of the coefficient of variation in our simulations did not exceed $4 \%$. However, variance indeed diverged from the deterministic approximation for genes under weaker selection $\left(s_{\text {het }} \lesssim 0.02\right)$. Figure $\mathbf{1}(\mathbf{a})$ also shows that the mean, $\mathrm{E}[k]$, is unaffected by genetic drift within the analyzed range of parameters.

\section{Incorporation of genetic drift in the selection inference}

Having established the effects of genetic drift on the population PTV allele frequency, we could then address the resulting effects on selection inference. Based on the approach described in Cassa et al. (2017), we estimated the parameters $\boldsymbol{\theta}$ of the distribution of heterozygous selection coefficients, 
$P\left(s_{\text {het }} ; \boldsymbol{\theta}\right)$, from fitting the observed distribution of per-gene PTV counts,

$$
P(k ; \boldsymbol{\theta}, n, U)=\int \mathrm{d} s_{\text {het }} P\left(s_{\text {het }} ; \boldsymbol{\theta}\right) P\left(k \mid s_{\text {het }} ; n, U\right),
$$

where $P\left(k \mid s_{\text {het }} ; n, U\right)$ denotes the conditional probability of observing count $k$ given $s_{\text {het }}$. At mutationselection balance, $P\left(k \mid s_{\text {het }} ; n, U\right)=\operatorname{Pois}\left(k ; n U / s_{\text {het }}\right)$, with $\operatorname{Pois}(k ; \lambda)$ denoting the Poisson distribution of $k$ with parameter $\lambda$.

Here, we fully accounted for the effects of drift through incorporation of the allele frequency spectrum, $\phi\left(X ; s_{\text {het }}, U\right)$. In the deterministic case, $X$ is assumed to be fixed at its expected value $U / s_{\text {het }}$ for a gene with mutation rate $U$ and under selection $s_{\text {het }}$. In contrast, we now explicitly include its variability:

$$
P\left(k \mid s_{\mathrm{het}} ; n, U\right)=\sum_{X} \operatorname{Pois}(k ; n X) \phi\left(X ; s_{\mathrm{het}}, U\right) .
$$

Population allele frequency spectra $\phi\left(X ; s_{\text {het }}, U\right)$ under the described demography were produced from simulations for a dense grid of mutation rates and selection strengths (Methods). We maximized the likelihood of the data over parameters $\boldsymbol{\theta}$, fitting the model in Eq. 1 to the observed distribution of $k$ in the ExAC NFE subsample (Supplementary Figure 2). As we found previously, the inverse Gaussian distribution provided the best parametric form for the fit to the distribution $P\left(s_{\text {het }} ; \boldsymbol{\theta}\right)$. To obtain gene-specific estimates of selection, $\hat{s}_{\text {het }}$, we derived the mean of the posterior distribution (Methods). Supplementary Table 1 contains the $\hat{s}_{\text {het }}$ values from the NFE subpopulation for the drift-inclusive and the deterministic case, including $95 \%$ posterior probability intervals.

We used the resulting per-gene estimates to understand the impact of a full demographic model relative to the deterministic estimates of heterozygous selection coefficients obtained under mutationselection balance. Figure 1(b) shows the direct comparison between the two scenarios for the NFE subset. Overall, the incorporation of drift in the model did not substantially change relative ranks of genes (Spearman rank correlation coefficient $=0.995$; Supplementary Figure 3). Estimates for genes under moderate to strong selection $\left(s_{\text {het }}>0.01, \mathrm{~N}=10,744\right.$ genes, $\left.66 \%\right)$ are very close to the deterministic estimates, only showing a slight downward shift on average. As variance due to genetic drift partly absorbs the variance of the prior distribution of selection coefficients, the latter decreased (by about 18\%). Genes under weaker selection $\left(s_{\text {het }}<0.01, \mathrm{~N}=5,535\right.$ genes, $34 \%$ ) appeared with largely the same rank, but showed a monotonic increase in estimated values of het- 
erozygous selection. The apparent convergence of selection coefficients to $\sim 0.004$ for these genes can be explained by the effects of genetic drift on the conditional probability in Eq. 2. For weak negative selection, $P\left(k \mid s_{\text {het }} ; n, U\right)$ becomes nearly flat in the regime of very small $s_{\text {het }}$, moving the estimates closer to the genome-wide mean.

We conclude that even though human demographic history is complex, a realistic model of recent population expansion suggests that, owing to their deleteriousness, the evolution of PTVs can be largely described in a deterministic framework.

\section{Test for differences between subpopulations}

As an additional way to test the utility and limits of the deterministic approximation to estimate selection, we used a data-driven approach. This approach relied on a comparison of PTV counts between different human subpopulations, free from assumptions made in simulations, such as panmixia. For a given gene, if the same selection coefficient $s_{\text {het }}$ acts on heterozygous PTVs in two subpopulations, we expect $\mathrm{E}\left[X_{1}\right]=\mathrm{E}\left[X_{2}\right]=U / s_{\text {het }}$. Here $X_{i}$ denotes the cumulative PTV allele frequency in subpopulation $i$. The PTV allele count in subsample $i$ was modeled as $k_{i} \sim \operatorname{Pois}\left(n_{i} U / s_{\text {het }}\right)$, where $n_{i}$ is the number of chromosomes sampled.

We tested whether this deterministic approximation was violated by comparing the Non-Finnish European (NFE, $i=1$ ) and African (AFR, $i=2$ ) ExAC subsamples using the C-test (Przyborowski and Wilenski, 1940). Given $k_{1}+k_{2} \equiv k$, the distribution of $k_{1}$ conditional on the total count $k$ is binomial with success probability $p=n_{1} /\left(n_{1}+n_{2}\right)$. We then computed the two-sided binomial pvalue of the observed value of $k_{1}$ for all genes in the set. Since the $k_{1}$ are discrete random variables, their p-values are not uniformly distributed. Therefore, in order to account for multiple testing, we compared the observed genome-wide distribution of binomial p-values to the p-value distribution obtained from a simulation under the null assumption. We generated 500 instances of simulated binomial PTV counts for each gene and computed the false discovery rate (FDR) conservatively, as the fraction of genes that is expected by chance to have a p-value equal to or less than a certain threshold. We then measured the number of genes with FDR below $5 \%, x_{\text {sig. }}$.

Table 1 shows the results for different intervals of the deterministic $s_{\text {het }}$ estimates, as well as the total number of genes in each interval, $x_{\text {tot }}$, and significant fraction. We found that a total of 870

out of 15,865 tested genes (5.5\%) show a significant deviation from the assumption of Poisson distributed counts with equal expected values in the two subpopulations. Of all 870 significant genes, $49 \%$ have $\hat{s}_{\text {het }} \leq 0.01$. Unlike our simulations, this data-driven approach is not expected to 
be contingent on assumptions about demographic structure or aspects of the population history.

\section{Prediction of de novo fraction using $\hat{s}_{\text {het }}$}

Some of the PTVs detected in a sample are de novo mutations rather than segregating alleles inherited from the parental generation. With increasing strength of negative selection, the population allele frequency, and thus the chance of inheriting a deleterious allele, is reduced and more of the observed deleterious mutations arise de novo. The fraction of de novo out of all PTVs equals $s_{\text {het }}$ for genes under negative selection in the deterministic limit. As shown in Methods, this result is also valid across a wide range of parameters at mutation-selection-drift balance (Supplementary Figure 4).

We collected de novo and inherited PTVs in autism-spectrum disorder (ASD) probands from about 4,000 parent-child trios (Kosmicki et al., 2017). For each gene, we computed the observed fraction

of de novo PTVs, $\hat{f}$, and compared it to the deterministic estimate of the heterozygous selection coefficient, $\hat{s}_{\text {het }}$. This analysis provides another independent and data-driven approach to test the validity of the $s_{\text {het }}$ estimates. Figure 2 shows the observed relation between $\hat{s}_{\text {het }}$ and $\hat{f}$. We find good agreement between $\hat{s}_{\text {het }}$ and $\hat{f}$ across a wide range of selection coefficients $\left(\hat{s}_{\text {het }} \geq 0.002\right)$. We repeated this analysis for the deterministic $s_{\text {het }}$ estimates obtained from the entire ExAC dataset (Cassa et al., 2017), which delivered comparable results (Supplementary Figure 5).

\section{Comparison of $\hat{s}_{\text {het }}$ to other measures of protein constraint}

Beyond the theoretical importance, evaluating selection on deleterious PTVs has practical applications in human genetics. Population-based measures of constraint, such as pLI (Lek et al., 2016) and RVIS (Petrovski et al., 2013), have been successfully used to prioritize genes in studies of neuropsychiatric and other diseases (Gussow et al., 2016). pLI measures the probability of a gene to be loss-of-function intolerant (Lek et al., 2016). This measure is based on a classification of genes into three categories and returns high values for genes under strong selection. By construction, this approach has limited resolution within this class of genes. Point estimation of $s_{\text {het }}$ characterizes the fitness loss beyond the binary classification of whether or not the gene is under constraint. Therefore, $\hat{s}_{\text {het }}$ has an advantage as a proxy for penetrance, disease age of onset and severity. This is illustrated in Figure 3, together with a comparison of the predictive power of pLI of the fraction of de novo mutations (see also Supplementary Figure 6). 


\section{Discussion}

The existence of stochastic effects in finite populations has long been known (Wright, 1931), predating diffusion theory approaches to the evolutionary dynamics of populations (Kimura, 1964). However, the impact of genetic drift on the fate of a newly arising mutation was not always appreciated. Partially due to historically insufficient amounts of data, the deterministic forces of mutation and selection were often the focus of population genetics analyses, corresponding to working in the limit of infinite effective population size, $N_{\mathrm{e}}$. The equilibrium state in that case is mutationselection balance. Arguably, a locus evolving under strong selection may still be considered in mutation-selection balance even if the effective population size is finite (that is, if it is large enough to ensure $4 N_{\mathrm{e}} s_{\text {het }} \gg 1$ ). In our earlier work, we hypothesized that also selection against heterozygous protein-truncating variants scaled by $N_{\mathrm{e}}$ would be sufficiently strong to ensure the assumption of mutation-selection balance (Cassa et al., 2017).

Here we have investigated the full effects of drift using simulations of a realistic human demography. This approach models recurrent mutations, is gauged by the observed NFE data in the ExAC cohort, and does not make assumptions about equilibrium. As a result of our analysis, we found that the deterministic mutation-selection balance approximation for counts of rare deleterious PTVs is applicable for genes under strong to intermediate selection, including genes with the previously inferred global mean of $\hat{s}_{\text {het }} \approx 0.06$. Selection estimates for this class of genes are highly robust to the incorporation of drift effects. For genes under relatively weak selection, the deterministic $s_{\text {het }}$ estimates provide a stable ranking useful as prioritization scores for practical applications in human genetics (Cassa et al., 2017).

The ExAC dataset used to estimate the deterministic $s_{\text {het }}$ given in Cassa et al. (2017) is composed of different subpopulations. Here we focused on the largest subset, NFE, with its established demographic dynamics, to compare deterministic evolution of PTVs to a scenario under genetic drift. Population structure can cause an increase in the variance of allele frequency. In the case of lethal but highly recessive variants, the mean allele frequency can even be reduced below the deterministic expectation of the combined population (Wright, 1937; Nei, 1968; Glemin, 2005). To address the effects of population structure on the deterministic $s_{\text {het }}$ estimates, we conducted two data-driven tests. First, we tested whether the PTV allele counts in the Non-Finnish European and the African ExAC subsamples were compatible with being generated under the same deterministic Poisson model with identical selection strength. Second, we analyzed the relationship between our estimates of heterozygous selection coefficients based on the combined ExAC dataset and the 
experimentally determined fractions of de novo mutations (Supplementary Figure 5). Both of these analyses are consistent with the simulation results, suggesting that the mutation-selection balance approximation is applicable to strongly to moderately selected genes.

\section{Materials and Methods}

\section{Protein-truncating variants in the NFE subsample}

In this analysis, we used the Non-Finnish European subsample from the ExAC data set version 0.3 (Lek et al., 2016), a set of jointly called exomes from 33,370 individuals ascertained with no known severe, early-onset Mendelian disorders. The mean coverage depth was calculated for each gene (canonical transcript from Ensembl v75, GENCODE v19) in the ExAC data set (mean 57.75; s.d. 20.96). Genes with average coverage depth of at least 30X were used in further analyses $(\mathrm{N}=17,199)$. Single nucleotide substitution variants annotated as PASS quality with predicted functional effects in the canonical transcript of 'stop_gained', 'splice_donor', or 'splice_acceptor' (as annotated by Variant Effect Predictor) were included in the analysis. Variants such as indels, in-frame mutations, and frameshift variants were excluded from this analysis, as many of these variants may have annotation issues or may not be functionally impactful. Along the same lines, we are mindful that not all PTVs will result in the same effect on gene function, due, for example, to alternative transcripts or the position dependence of nonsense-mediated decay (Rivas et al., 2015). To address this, variants were filtered using LOFTEE and restricted to those predicted with high confidence to have consequences in the canonical transcript.

For each of the 17,199 genes, we have observable values for $(k, n, U)$, where $k$ denotes the total number of observed PTV alleles in the NFE population sample of $n$ chromosomes covered in the gene, and $U$ the PTV mutation rate across the canonical gene transcript from a mutational model (Samocha et al., 2014; Francioli et al., 2015). Values of $U$ for each gene from Samocha et al. (2014) were used along with the number of well-covered chromosomes $n$ in each gene to generate the null mutational expectation of neutral evolution, $n U$. Incorrectly specified values from this mutational model could alter estimates of selection for individual genes, as higher estimates of selection are made in genes with greater depletions from the null expectation model. Our inference of selection coefficients relies on the assumption that the cumulative population frequency of PTV mutations, $X$, is small owing to strong negative selection, so genes with $\hat{X}=k / n>0.001$ are omitted from the analysis, leaving 16,279 genes in the NFE subsample. On these genes, there are 115,651 NFE PTVs in total with a mean PTV count of 7.1, while the maximum is 66. Supplementary Table 1 
contains the values $k, n$ and $U$ for all 16,279 tested genes in the NFE subsample.

\section{De novo and transmitted PTVs in an ASD trio sequencing cohort}

For the derivation of the de novo fraction, we downloaded 5,856 de novo variants from 3,982 individuals ascertained for autism-spectrum disorder from Kosmicki et al. (2017). We also incorporated 72 published, validated ASD de novo variants from Krumm et al. (2015) and 119 ASD de novo coding variants from Werling et al. (2018), bringing the total number of de novo variants to 6,047 for ascertained individuals with ASD. One pair of the published ASD probands was a duplicate (10C112515 and 14621.p1), so we removed one of them (10C112515), which reduced the total number of individuals ascertained for ASD from 3,982 to 3,981 and their total number of de novo variants to 6,044. Lastly, following the protocol from The Deciphering Developmental Disorders Study (2017), we restricted the number of de novo variants to one variant per person, per gene, prioritizing the de novo variant with the most severe consequence, which removed 64 ASD de novo variants, bringing the final total to 5,980 ASD de novo variants. To restrict to PTVs, we included all variants with annotations 'stop_gained', 'splice_donor_variant', or 'splice_acceptor_variant' (331 variants).

For the transmission analysis of segregating variants, we used all PTV variants with PASS quality in at least $95 \%$ of all 4,319 sequenced trios from the Autism Sequencing Consortium freeze v13 (see Kosmicki et al. (2017)). This delivered a total of 22,666 distinct variants found in the parents, which were either transmitted or untransmitted to the probands ascertained for ASD. Because of the difference of 338 tested trios between the de novo and transmission datasets, we weighted each PTV category correspondingly for the estimation of the de novo fraction $\hat{f}$. For the NFE dataset

with 16,279 genes with $\hat{X}=k / n \leq 0.001$, the de novo PTVs were located on 265 genes, while the parental segregating mutations were distributed across 8,092 genes. We used the set of 6,203 genes with at least one PTV (transmitted or de novo) in the comparison with the $s_{\text {het }}$ estimates. In case of $s_{\text {het }}$ estimated from the entire ExAC dataset, of the 15,998 genes with $\hat{X}=k / n \leq 0.001$ (Cassa et al., 2017), 261 had de novo PTVs and 7,861 had segregating variants, and 6,000 genes with at least one mutation (transmitted or de novo) were tested.

\section{Analytical derivation of PTV count variance}

For most genes, protein-truncating alleles are both individually and collectively rare. We define the cumulative PTV allele frequency of a gene in the population, $X=\sum_{j} x_{j}$, where the sum is over all 
PTV sites $j$ on the gene with respective allele frequencies $x_{j}$. This is motivated by the simplifying assumption of identical selection coefficients for all PTVs within a gene and the observation that the frequency of the vast majority of PTVs is extremely low, such that the occurrence of multiple variable sites within a gene on a single haplotype is also extremely low $\left(2 n x_{j} x_{k}<1\right.$ for sample size $n)$.

The frequency $X$ is governed by the demographic dynamics of the population. For heterozygous selection coefficient $s_{\text {het }}$ and cumulative mutation rate $U$, it follows the allele frequency distribution $\phi\left(X ; s_{\text {het }}, U\right)$. Given frequency $X$ in the population, we expect to see on average $\mathrm{E}[k]=n X$ mutations in a sample of $n$ chromosomes, hence we arrive at Eq. 2,

$$
\begin{aligned}
P\left(k \mid s_{\text {het }} ; n, U\right) & =\sum_{X} P(k \mid X ; n) P\left(X \mid s_{\text {het }} ; U\right) \\
& \approx \sum_{X} \operatorname{Pois}(k ; n X) \phi\left(X ; s_{\text {het }}, U\right) .
\end{aligned}
$$

Here, Poisson sampling with parameter $n X$ again represents the limit of binomial sampling for small success probabilities $x_{j}$ and large sample size $n$. In particular, we are interested in how genetic drift affects the mean and variance of the PTV count $k$. For a given selective effect $s_{\text {het }}$ we obtain the expected value of $k$ from Eq. 2,

$$
\mathrm{E}[k]=n \mathrm{E}[X]
$$

while the variance is given by

$$
\operatorname{Var}[k]=n \mathrm{E}[X]+n^{2} \operatorname{Var}[X]
$$

\section{Analytical approximation for strong selection on heterozygous sites}

Both $\mathrm{E}[X]$ and $\operatorname{Var}[X]$ depend on the evolutionary dynamics of the population, but it is instructive to compare to the equilibrium case of strong purifying selection on heterozygous variation described by Nei (1968). Nei showed that in that case the theoretical allele frequency distribution can be ap-

proximated by a gamma distribution with shape parameter $4 N_{\mathrm{e}} U$ and scale parameter $1 /\left(4 N_{\mathrm{e}} s_{\text {het }}\right)$, where $N_{\mathrm{e}}$ again denotes the effective population size. Under this assumption, Eqs. 3 and 4 become

$$
\mathrm{E}_{\mathrm{Nei}}[k]=n \frac{U}{s_{\text {het }}}
$$


and

$$
\begin{aligned}
\operatorname{Var}_{\mathrm{Nei}}[k] & =n \frac{U}{s_{\text {het }}}+n^{2}\left(\frac{U}{4 N_{\mathrm{e}} s_{\text {het }}^{2}}\right) \\
& =\frac{n U}{s_{\text {het }}}\left(1+\frac{n}{4 N_{\mathrm{e}} s_{\text {het }}}\right) .
\end{aligned}
$$

When we compare this to the analogous expressions for mean and variance under the deterministic assumption of mutation-selection balance,

$$
\mathrm{E}_{\mathrm{det}}[k]=\operatorname{Var}_{\operatorname{det}}[k]=\frac{n U}{s_{\text {het }}}
$$

we find the same expected value. For the excess variance we consequently expect to see a suppression with increasing effective size of the population from which the sample was drawn, as well as with increasing selection strength.

\section{Population genetics simulations}

To understand the interaction between mutation, selection, genetic drift, and population sampling, we wrote a custom Wright-Fisher simulator appropriate for the parameter regimes of interest. We assumed infinite recombination, such that all sites evolve independently, in a panmictic population with a single value of genic (additive) natural selection per simulation, and a fixed mutation rate with no back mutations. We modeled genes as biallelic sites with a mutation rate associated with the sum of all PTV targets in the gene, $U$. For each simulated biallelic site, recurrent mutations were Poisson sampled with mean $A U$, where $A$ is the number of ancestral, i.e. unmutated, chromosomes. For a given mutation rate and selection coefficient we generated 100,000 independent realizations, providing a distribution of biallelic frequencies. We simulated a dense grid of mutation rates $\left(U \in\left[10^{-7}, 7.16 \times 10^{-5}\right]\right)$ associated with the PTV-specific mutation rate estimates provided in Samocha et al. (2014). For each biallelic mutation rate, we simulated a range of 12 selection coefficients $\left(s_{\text {het }} \in\left[5 \times 10^{-6}, 1\right]\right)$.

We equilibrated simulations at the human ancestral population size of $N_{\mathrm{e}}=14,474$ for a burn-in period of $10 N_{\mathrm{e}}=144,740$ generations. At this point, we simulated 5,921 generations of European demographic history, following the parameters specified in Tennessen et al. (2012). This corresponds to a constant population size for 3,880 generations, then a 1,120 generation bottleneck down to $2 N_{\mathrm{e}}=3,722$ individuals, then a 716 generation mild exponential growth phase with a growth rate of 0.00307 , followed by a final exponential growth epoch of 205 generations. The final 
exponential growth phase was modeled three times with three distinct growth rates: growth = $\{0.0195,0.024,0.030\}$. The first two growth rates were chosen based on published values of the final European effective population size: 0.0195 from Tennessen et al. (2012) and 0.024 roughly corresponding to Gao and Keinan (2014), corresponding to final population sizes of around $N_{\mathrm{e}}=0.5$ million and $N_{\mathrm{e}}=1.25$ million, respectively. We estimated the final growth rate by simulating neutral variation through a dense range of growth rates and comparing to observations of the relative number of singletons to all segregating sites for synonymous sites in the ExAC NFE sample. This comparison was performed using a mutation rate of $3.8 \times 10^{-9}$ for non-CpG transversions (Kong et al., 2012), and comparing to the fraction of non-CpG transversion singletons in the NFE sample. CpG and non-CpG transitions were explicitly ignored for these purposes, as they are known to exhibit the effects of recurrent mutations in the ExAC sample due to elevated mutation rates relative to non-CpG transversions. An effective population size growth rate of 0.03 per generation in the last exponential epoch provided a fraction of singletons of 0.636 , which is highly consistent with the number of observed singletons in ExAC NFE of 0.633. Unsurprisingly, the final population size of $N_{\mathrm{e}}=4.3$ million found by matching the fraction of singletons is consistent with a recent population size inference performed on the same dataset (Harpak et al., 2016). We are mindful that we cannot exclude the effects of selection at linked sites affecting synonymous variants in the ExAC NFE sample. We simulated all mutation rate and selection strength combinations through all three European demographies, and found a distribution of biallelic frequencies (including the number of monomorphic sites) for each. The distributions corresponding to the demography matching the ExAC NFE dataset (final growth rate of 0.03) were used in all subsequent analyses.

\section{Assessment of variance in comparison to Poisson sampling variance}

To analyze the deviation from Poisson variance, we downsampled the final population of the simulation to the sample size of the NFE individuals in ExAC. The variance was computed over independent sites and compared to the theoretical mean of the Poisson distribution to assess the effects of genetic drift on the variance for a gene relative to the Poisson variance expected at mutation-selection balance.

Figure 1(a) shows the deviation of the coefficient of variation from the Poisson expectation due to the effects of a realistic demographic history (growth rate of 0.030, consistent with ExAC NFE ob-

servations). Simulations are shown for the non-CpG transversion mutation rate of $3.8 \times 10^{-9}$, as the inflation of the Poisson assumption should be independent of the mutation rate. Supplementary 
Figure 1 shows the same deviation in all three demographic models, suggesting that the slowest growth model (Tennessen et al., 2012) inferred from 1,351 European American exomes deviates at higher selection strengths than the demographic model associated with the much larger ExAC dataset. However, all three demographies are qualitatively consistent in their behavior, suggesting the departure from the Poisson assumption (variance to mean ratio $>1.5$ ) occurs at roughly $s_{\text {het }}=$ $0.02,0.03$, and 0.06 for growth rates of $0.030,0.024$, and 0.0195 , respectively.

\section{Hierarchical model to estimate $P\left(s_{\text {het }} ; \boldsymbol{\theta}\right)$}

From Eqs. 1 and 2 we derive the expression for the PTV count distribution when $s_{\text {het }}$ is drawn from $P\left(s_{\text {het }} ; \boldsymbol{\theta}\right)$ with parameter vector $\boldsymbol{\theta}$ :

$$
P(k ; \boldsymbol{\theta}, n, U)=\int \mathrm{d} s_{\text {het }} P\left(s_{\text {het }} ; \boldsymbol{\theta}\right) \sum_{X} \operatorname{Pois}(k ; n X) \phi\left(X ; s_{\text {het }}, U\right) .
$$

At mutation-selection balance, $X_{\text {eq }}=U / s_{\text {het }}$ and $\phi\left(X ; s_{\text {het }}, U\right)=\delta_{X, U / s_{\text {het }}}$, where $\delta$ denotes the Kronecker delta, yielding

$$
P(k ; \boldsymbol{\theta}, n, U)=\int \mathrm{d} s_{\text {het }} P\left(s_{\text {het }} ; \boldsymbol{\theta}\right) \operatorname{Pois}\left(k ; n U / s_{\text {het }}\right)
$$

which exactly reproduces Eq. 4 of Cassa et al. (2017).

\section{Fit to the PTV count distribution using simulations of a realistic demography}

To evaluate the differences between the selection inference under the original mutation-selection balance assumption, Eq. 9, and the full model incorporating genetic drift, we applied Eq. 8 to fit the observed distribution of per-gene PTV counts. Because of the complex demographic histories of the different subpopulations constituting the ExAC dataset, particularly substantial recent population expansions, the simple equilibrium approximation by Nei (1968) is not sufficient to substitute for $\phi\left(X ; s_{\text {het }}, U\right)$. Instead, we used the population allele frequency spectrum obtained from forward simulations described above, based on published demographies for the largest subpopulation, NonFinnish Europeans. Since $\phi\left(X ; s_{\text {het }}, U\right)$ depends on $s_{\text {het }}$, we used the simulated allele frequency spectra for each of 12 discrete values spanning six orders of magnitude: $s_{\text {het }}=\left\{5 \times 10^{-6}, 1.6 \times\right.$ $\left.10^{-5}, 5 \times 10^{-5}, 1.6 \times 10^{-4}, 5 \times 10^{-4}, 1.6 \times 10^{-3}, 5 \times 10^{-3}, 1.6 \times 10^{-2}, 5 \times 10^{-2}, 1.6 \times 10^{-1}, 5 \times 10^{-1}, 1\right\}$, and rewrote the integral Eq. 8 as a sum. Genes were binned by their cumulative mutation rate $U$ and for each $s_{\text {het }}$ we simulated $\phi\left(X ; s_{\text {het }}, U\right)$ for $U$-bins of width $10^{-7}$ if $U<4 \times 10^{-6}$, of width 
$2 \times 10^{-7}$ for $4 \times 10^{-6}<U<1.4 \times 10^{-5}$ and for exact $U$ values above. Each spectrum contained 100,000 random instances of simulated frequencies $X$.

Parameters of the discretized and renormalized $P\left(s_{\text {het }} ; \boldsymbol{\theta}\right)$ were estimated from maximizing the likelihood $(\mathcal{L})$, as described in Cassa et al. (2017). We compared the fits from three different twoparameter functional forms for $P\left(s_{\text {het }} ; \boldsymbol{\theta}\right)$ ( $-\log \mathcal{L}$ in brackets): inverse Gaussian (48417.4), gamma (48419.0), and inverse gamma distribution (48477.3). As for the deterministic scenario, we found that the highest likelihood model is the inverse Gaussian distribution,

$$
\operatorname{IG}\left(s_{\text {het }} ; \boldsymbol{\theta}=(\alpha, \beta)\right)=\sqrt{\frac{\beta}{2 \pi s_{\text {het }}^{3}}} \mathrm{e}^{-\frac{\beta\left(s_{\text {het }}-\alpha\right)^{2}}{2 s_{\text {het }} \alpha^{2}}},
$$

with mean $\alpha$ and shape parameter $\beta$. For comparison, we also give the inferred parameters of the gamma distribution, $\operatorname{Gamma}\left(s_{\text {het }} ; a, b\right)=\frac{1}{b^{a} \Gamma(a)} \mathrm{e}^{-\frac{s_{\text {het }}}{b}} s_{\text {het }}^{a-1}$, with estimated shape parameter $a=0.406$ and scale parameter $b=0.099$. Because the tested distributions are defined on the positive real axis, but the heterozygous selection coefficient $s_{\text {het }} \leq 1, P\left(s_{\text {het }} ; \boldsymbol{\theta}\right)$ was truncated at $s_{\max }=2.5$, corresponding to a probability density of $<0.01$. We find a slight dependence of the inferred parameters $\hat{\boldsymbol{\theta}}$ on $s_{\max }\left(\overline{\hat{\alpha}}=0.061 \pm 0.004, \overline{\hat{\beta}}=0.0070 \pm 0.0005 ; s_{\max } \in\{1,1.5,2,2.5\}\right)$, while the rank correlation changes by less than $0.1 \%$. Supplementary Figure 2 shows the resulting fit, $P\left(s_{\text {het }} ; \hat{\boldsymbol{\theta}}\right)$, in comparison to the fit obtained under the mutation-selection balance assumption. As expected, the incorporation of genetic drift entails a moderate shrinkage of the variance (by 18\%), as under the deterministic assumption its effects on PTV count variance are partly absorbed by $P\left(s_{\text {het }} ; \hat{\boldsymbol{\theta}}\right)$. Per-gene estimates were derived as the mean of the posterior distribution:

$$
\hat{s}_{\text {het }}=\frac{1}{P(k ; \hat{\boldsymbol{\theta}}, n, U)} \int \mathrm{d} s_{\text {het }} s_{\text {het }} P\left(s_{\text {het }} ; \hat{\boldsymbol{\theta}}\right) \sum_{X} \operatorname{Pois}(k ; n X) \phi\left(X ; s_{\text {het }}, U\right) .
$$

Supplementary Table 1 provides the $s_{\text {het }}$ estimates as well as the $95 \%$ credible intervals derived from the posterior distribution. In case of the drift-inclusive estimates, these intervals are constrained to the 12 logarithmically discretized values of $s_{\text {het }}$ used in the inference.

\section{Relationship between $s_{\text {het }}$ and the de novo fraction of PTVs}

In the limit of strong selection and at mutation-selection balance, the expectation for the cumulative PTV population allele frequency on a gene is $U / s_{\text {het }}$. The relative fitness of an individual with a segregating PTV mutation in the current generation is $1-s_{\text {het }}$, rendering the probability for a 
transmitted PTV in the next generation

$$
\operatorname{Pr}(\text { transmitted PTV }) \approx \frac{2 U}{s_{\text {het }}}\left(1-s_{\text {het }}\right)
$$

where we assumed $U \lesssim 10^{-6} \ll 1$ and the strong selection approximation $U / s_{\text {het }}<10^{-3} \ll 1$. The probability for a de novo PTV mutation to occur is

$$
\operatorname{Pr}(\text { de novo } \mathrm{PTV}) \approx 2 U
$$

From this, we compute the fraction of de novo among all PTVs in the limit of strong selection:

$$
f=\frac{\operatorname{Pr}(\text { de novo } \mathrm{PTV})}{\operatorname{Pr}(\text { de novo } \mathrm{PTV})+\operatorname{Pr}(\text { transmitted } \mathrm{PTV})} \approx s_{\text {het }} .
$$

More generally, we can derive the fraction of de novo mutations from the equilibrium allele frequency spectrum for any value of $s_{\text {het }}$. At mutation-selection-drift balance and in the limit of small mutation rates, the derived allele frequency spectrum is approximately given by

$$
\phi_{\text {eq }}\left(X ; s_{\text {het }}, U, N_{\mathrm{e}}\right)=\frac{1-\mathrm{e}^{4 N_{\mathrm{e}} s_{\mathrm{het}}(1-X)}}{1-\mathrm{e}^{4 N_{\mathrm{e}} s_{\mathrm{het}}}} \frac{4 N_{\mathrm{e}} U}{X(1-X)} .
$$

Hence, we obtain for the probability of inheritance

$$
\begin{aligned}
\operatorname{Pr}(\text { transmitted PTV }) & \approx\left(1-s_{\text {het }}\right) \int 2 X(1-X) \phi_{\text {eq }}\left(X ; s_{\text {het }}, U, N_{\mathrm{e}}\right) \mathrm{d} X \\
& =\left(1-s_{\text {het }}\right)\left(\frac{2 U}{s_{\text {het }}}+\frac{8 N_{\mathrm{e}} U}{1-\mathrm{e}^{4 N_{\mathrm{e}} s_{\mathrm{het}}}}\right) .
\end{aligned}
$$

Supplementary Figure 4 shows the resulting expression for the de novo fraction as a function of $s_{\text {het }}$. Based on our estimates of recent effective population size, which is the most relevant for rare deleterious mutations, on the order of millions, we expect the one-to-one correlation between $s_{\text {het }}$ and $f$ to hold across the largest part of the selection regime. This renders the de novo fraction of PTV alleles an excellent cross-check for the $s_{\text {het }}$ estimates.

\section{Acknowledgements}

We kindly thank Brian Charlesworth and William Hill for their interest in our work and for inspiring this study. We would like to thank Jeremy Berg, Guy Sella, Molly Przeworski and Alexey Kondrashov for their helpful suggestions and feedback. This work was supported by the National 
Institutes of Health (GM127131, MH101244, HG009088).

\section{References}

Browning, S. R. and Browning, B. L. (2015). Accurate non-parametric estimation of recent effective population size from segments of identity by descent. The American Journal of Human Genetics, 97(3):404-418.

Bürger, R., Wagner, G. P., and Stettinger, F. (1989). How much heritable variation can be maintained in finite populations by mutation-selection balance? Evolution, 43(8):1748-1766.

Cassa, C. A., Weghorn, D., Balick, D. J., Jordan, D. M., Nusinow, D., Samocha, K. E., O’DonnellLuria, A., MacArthur, D. G., Daly, M. J., Beier, D. R., et al. (2017). Estimating the selective effects of heterozygous protein-truncating variants from human exome data. Nature Genetics, 49(5):806.

Cassa, C. A., Weghorn, D., Balick, D. J., Jordan, D. M., Nusinow, D., Samocha, K. E., ODonnellLuria, A., MacArthur, D. G., Daly, M. J., Beier, D. R., et al. (2019). Reply to selective effects of heterozygous protein-truncating variants. Nature Genetics, 51(1):3.

Charlesworth, B. and Hill, W. G. (2019). Selective effects of heterozygous protein-truncating variants. Nature Genetics, 51(1):2.

Do, R., Balick, D., Li, H., Adzhubei, I., Sunyaev, S., and Reich, D. (2015). No evidence that selection has been less effective at removing deleterious mutations in europeans than in africans. Nature Genetics, 47(2):126.

Fisher, R. A. (1930). The genetical theory of natural selection: a complete variorum edition. Oxford University Press.

Francioli, L. C., Polak, P. P., Koren, A., Menelaou, A., Chun, S., Renkens, I., Van Duijn, C. M., Swertz, M., Wijmenga, C., Van Ommen, G., et al. (2015). Genome-wide patterns and properties of de novo mutations in humans. Nature Genetics, 47(7):822.

Gao, F. and Keinan, A. (2014). High burden of private mutations due to explosive human population growth and purifying selection. BMC Genomics, 15(4):S3.

Glemin, S. (2005). Lethals in subdivided populations. Genetics Research, 86(1):41-51.

Gussow, A. B., Petrovski, S., Wang, Q., Allen, A. S., and Goldstein, D. B. (2016). The intolerance to functional genetic variation of protein domains predicts the localization of pathogenic mutations within genes. Genome Biology, 17(1):9.

Harpak, A., Bhaskar, A., and Pritchard, J. K. (2016). Mutation rate variation is a primary determinant of the distribution of allele frequencies in humans. PLoS Genetics, 12(12):e1006489.

Keinan, A. and Clark, A. G. (2012). Recent explosive human population growth has resulted in an excess of rare genetic variants. Science, 336(6082):740-743.

Kimura, M. (1964). Diffusion models in population genetics. Journal of Applied Probability, 1(2):177-232. 
Kong, A., Frigge, M. L., Masson, G., Besenbacher, S., Sulem, P., Magnusson, G., Gudjonsson, S. A., Sigurdsson, A., Jonasdottir, A., Jonasdottir, A., et al. (2012). Rate of de novo mutations and the importance of fathers age to disease risk. Nature, 488(7412):471.

Kosmicki, J. A., Samocha, K. E., Howrigan, D. P., Sanders, S. J., Slowikowski, K., Lek, M., Karczewski, K. J., Cutler, D. J., Devlin, B., Roeder, K., et al. (2017). Refining the role of de novo protein-truncating variants in neurodevelopmental disorders by using population reference samples. Nature Genetics, 49(4):504.

Krumm, N., Turner, T. N., Baker, C., Vives, L., Mohajeri, K., Witherspoon, K., Raja, A., Coe, B. P., Stessman, H. A., He, Z.-X., et al. (2015). Excess of rare, inherited truncating mutations in autism. Nature Genetics, 47(6):582.

Lande, R. (1976). Natural selection and random genetic drift in phenotypic evolution. Evolution, $30(2): 314-334$.

Lek, M., Karczewski, K. J., Minikel, E. V., Samocha, K. E., Banks, E., Fennell, T., ODonnell-Luria, A. H., Ware, J. S., Hill, A. J., Cummings, B. B., et al. (2016). Analysis of protein-coding genetic variation in 60,706 humans. Nature, 536(7616):285.

Nei, M. (1968). The frequency distribution of lethal chromosomes in finite populations. Proceedings of the National Academy of Sciences, 60(2):517-524.

Petrovski, S., Wang, Q., Heinzen, E. L., Allen, A. S., and Goldstein, D. B. (2013). Genic intolerance to functional variation and the interpretation of personal genomes. PLoS Genetics, 9(8):e1003709.

Przyborowski, J. and Wilenski, H. (1940). Homogeneity of results in testing samples from poisson series. Biometrika, 31(3/4):313-323.

Rivas, M. A., Pirinen, M., Conrad, D. F., Lek, M., Tsang, E. K., Karczewski, K. J., Maller, J. B., Kukurba, K. R., DeLuca, D. S., Fromer, M., et al. (2015). Effect of predicted protein-truncating genetic variants on the human transcriptome. Science, 348(6235):666-669.

Samocha, K. E., Robinson, E. B., Sanders, S. J., Stevens, C., Sabo, A., McGrath, L. M., Kosmicki, J. A., Rehnström, K., Mallick, S., Kirby, A., et al. (2014). A framework for the interpretation of de novo mutation in human disease. Nature Genetics, 46(9):944.

Tennessen, J. A., Bigham, A. W., Oconnor, T. D., Fu, W., Kenny, E. E., Gravel, S., McGee, S., Do, R., Liu, X., Jun, G., et al. (2012). Evolution and functional impact of rare coding variation from deep sequencing of human exomes. Science, 337(6090):64-69.

The Deciphering Developmental Disorders Study (2017). Prevalence and architecture of de novo mutations in developmental disorders. Nature, 542(7642):433.

Werling, D. M., Brand, H., An, J.-Y., Stone, M. R., Zhu, L., Glessner, J. T., Collins, R. L., Dong, S., Layer, R. M., Markenscoff-Papadimitriou, E., et al. (2018). An analytical framework for whole-genome sequence association studies and its implications for autism spectrum disorder. Nature Genetics, 50:727-736.

Williamson, S. H., Hernandez, R., Fledel-Alon, A., Zhu, L., Nielsen, R., and Bustamante, C. D. (2005). Simultaneous inference of selection and population growth from patterns of variation in the human genome. Proceedings of the National Academy of Sciences, 102(22):7882-7887.

Wright, S. (1931). Evolution in mendelian populations. Genetics, 16(2):97. 
Wright, S. (1937). The distribution of gene frequencies in populations. Proceedings of the National Academy of Sciences, 23(6):307-320. 
(a)

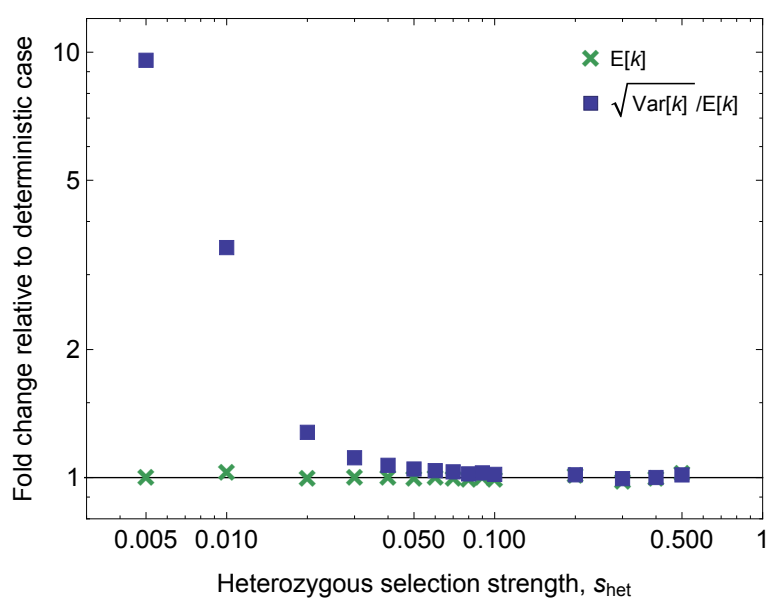

(b)

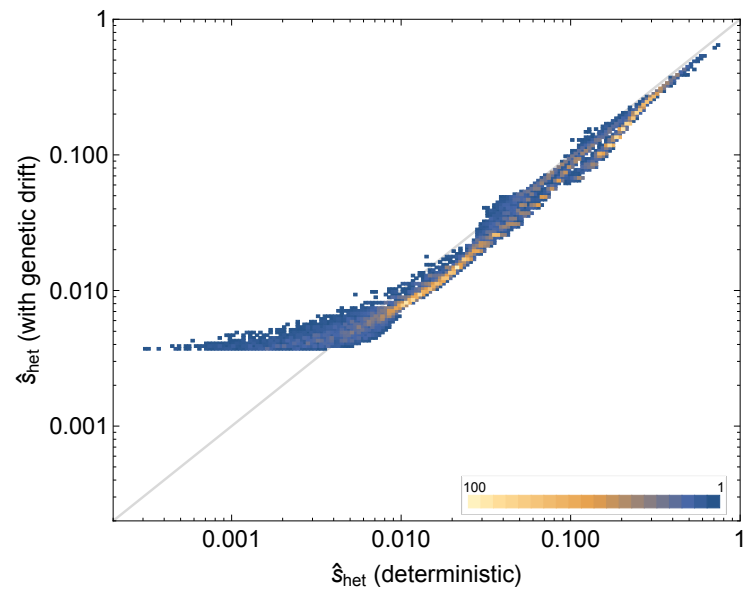

Figure 1: Comparison of the deterministic mutation-selection balance model with the model that includes the effects of genetic drift, in the NFE demography. (a) Fold change in the coefficient of variation (squares) and the mean (crosses) of the number of PTV mutations, $k$, relative to the deterministic case, obtained from simulations of a realistic demography of the ExAC NFE sample for different values of heterozygous selection strength $s_{\text {het }}$. (b) Heat map of gene-specific estimates for all 16,279 tested genes from the NFE sample, showing deterministic (x-axis) and drift-inclusive (y-axis) $s_{\text {het }}$ estimates. Note the double-logarithmic axes in both panels. 


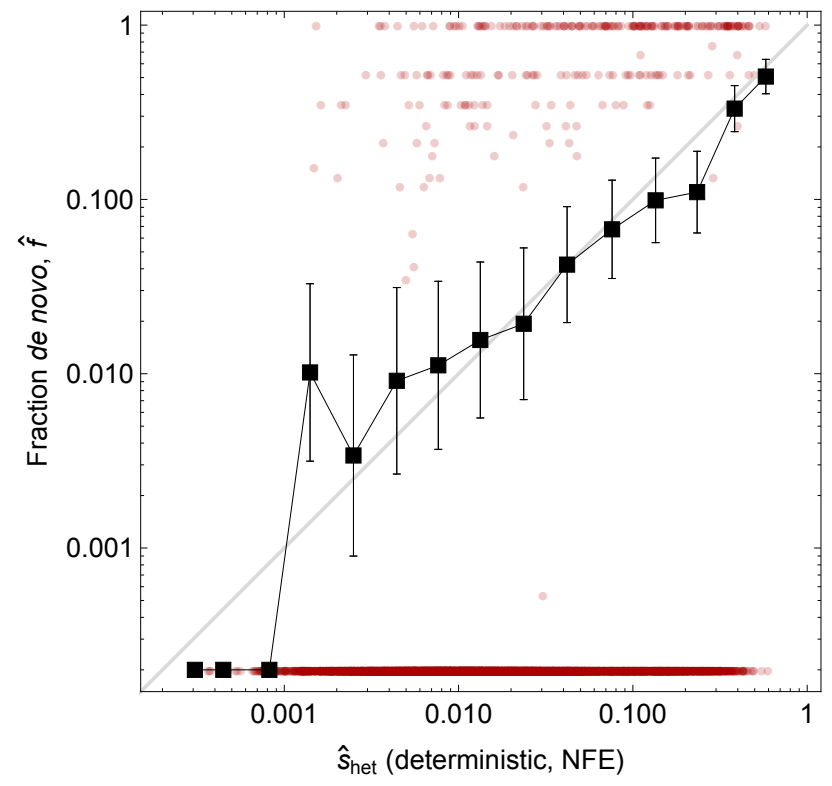

Figure 2: In the strong selection limit, $s_{\text {het }}$ is a predictor of the fraction of de novo PTVs, $f$. De novo fraction of PTV mutations was estimated for 6,203 (out of 16,279) genes with at least one PTV (de novo or transmitted) in an ASD cohort of about 4,000 parent-child trios (y-axis) and compared to the deterministic $\hat{s}_{\text {het }}$ derived from the NFE sample (x-axis). Red dots denote individual genes (genes with $\hat{f}=0$ were assigned $\hat{s}_{\text {het }}=2 \times 10^{-4}$ for illustration purposes). Black squares connected by black lines denote the mean in bins along the x-axis of logarithmic width $\Delta \log \left[\hat{s}_{\text {het }}\right]=0.25$ (number of genes per bin from left to right: $\{1,10,43,148,400,811,1117,1158$, $870,597,359,360,236,90,3\})$. Vertical error bars show the standard error of the mean per bin for $\hat{f}$. Corresponding error bars for $\hat{s}_{\text {het }}$ are smaller than the marker size. Grey line denotes the diagonal. 
(a)
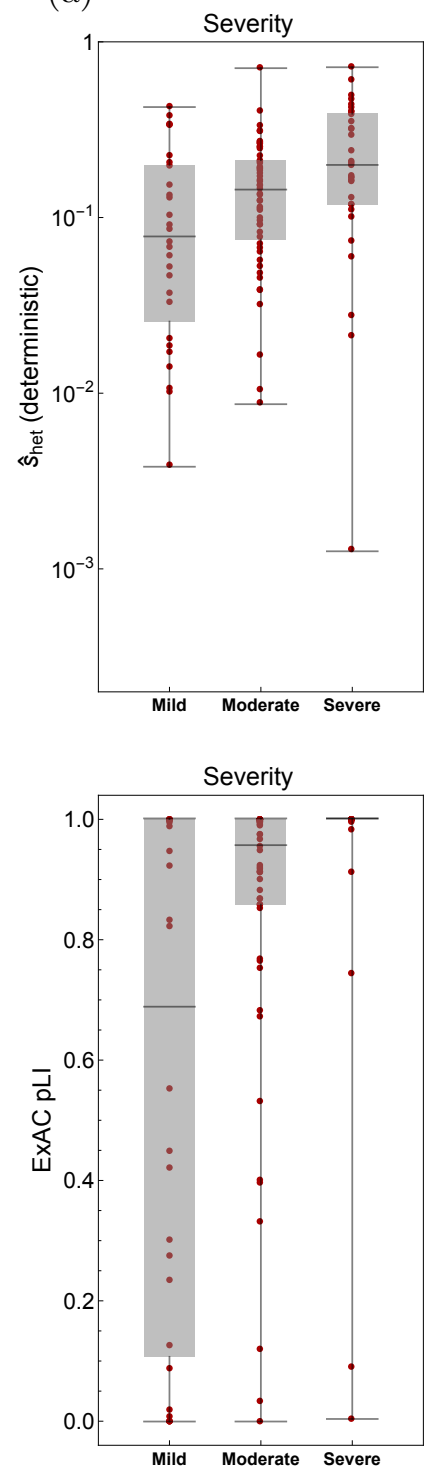

(b)
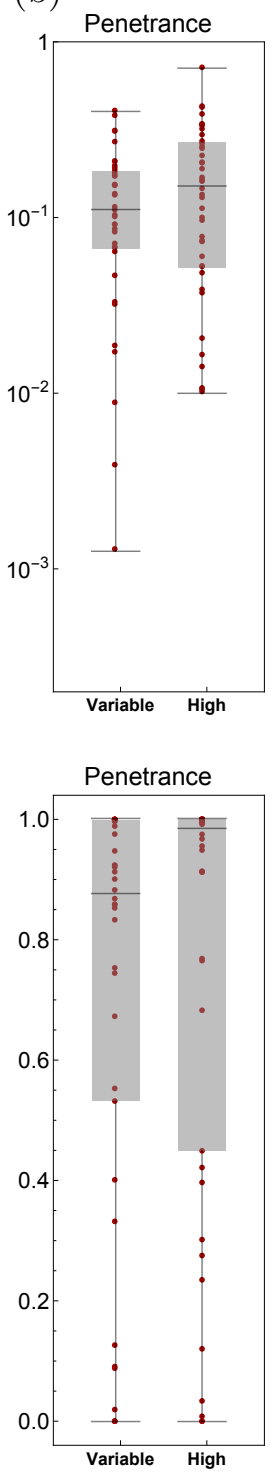

(c)
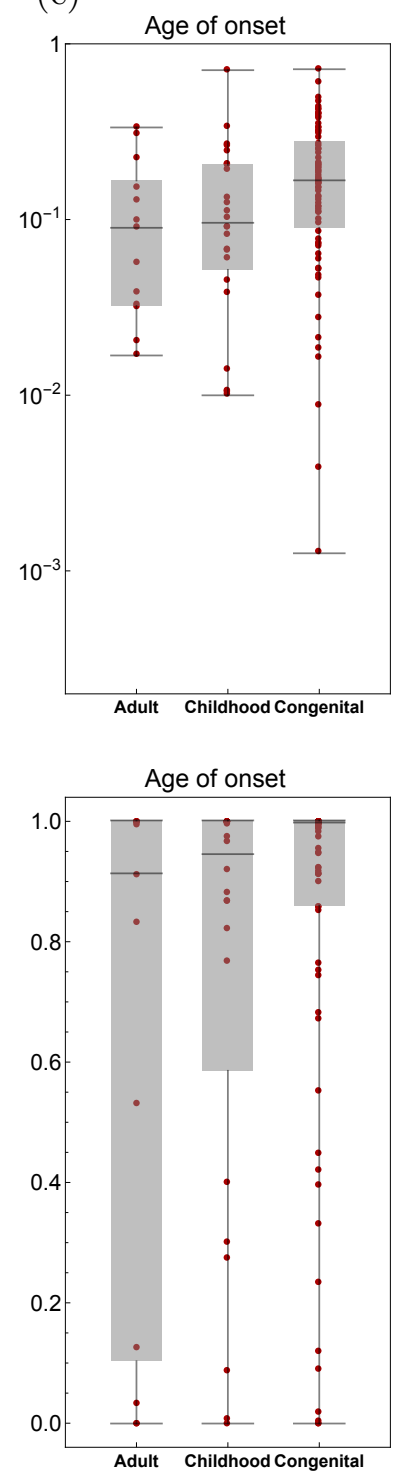

(d)
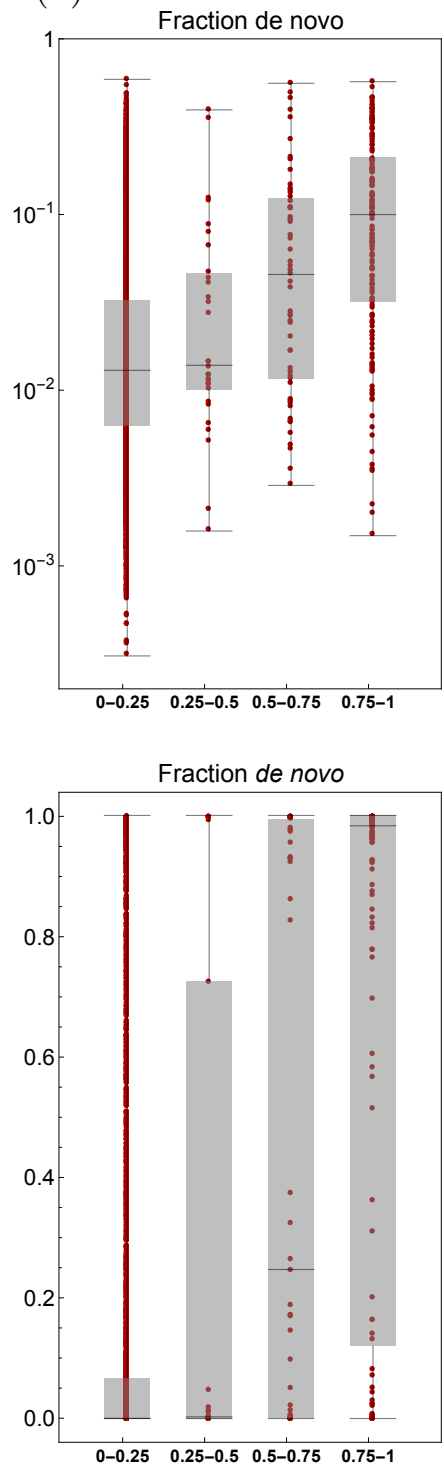

Figure 3: Comparison of per-gene selection estimates, $\hat{s}_{\text {het }}$, with a measure of probability of lossof-function intolerance, pLI (Lek et al., 2016). Shown is the correlation with independent measures of gene importance. (a-c) Data on disease severity, penetrance, and age of onset (x-axes) for a set of 113 haploinsufficient disease-associated genes of high confidence (ClinGen Dosage Sensitivity Project) were compared to deterministic NFE $\hat{s}_{\text {het }}$ (top row) and pLI (bottom row) predictions (y-axis). (d) The fraction of de novo PTVs $(\hat{f})$, shown in bins of width 0.25 for 5,930 genes with at least one transmitted or de novo PTV and a pLI annotation (x-axis), was derived from an ASD trio-sequencing dataset (top: NFE $\hat{s}_{\text {het }}$, bottom: pLI). Note that due to the small number of PTVs per gene in the ASD cohort, the distribution of $\hat{f}$ on the range $[0,1]$ is not smooth. Red dots denote individual genes, grey boxes enclose the central quartiles of the distribution in each category, and black horizontal bars through grey boxes show the median. Note the logarithmic y-axis in the top row, while the bottom row has a linear y-axis. 


\begin{tabular}{|r|r|r|r|}
\hline$\hat{s}_{\text {het }}$ interval & $x_{\text {sig }}$ & $x_{\text {tot }}$ & $x_{\text {sig }} / x_{\text {tot }}$ \\
\hline$[0.000,0.005]$ & 220 & 1690 & 0.124 \\
\hline$[0.005,0.010]$ & 202 & 2315 & 0.086 \\
\hline$[0.010,0.020]$ & 148 & 2744 & 0.052 \\
\hline$[0.020,0.050]$ & 140 & 3275 & 0.041 \\
\hline$[0.050,0.200]$ & 102 & 4050 & 0.029 \\
\hline$[0.200,0.500]$ & 58 & 1765 & 0.028 \\
\hline$[0.500,1.000]$ & 0 & 26 & 0.000 \\
\hline
\end{tabular}

Table 1: Fraction, $x_{\text {sig }} / x_{\text {tot }}$, of genes with significant, FDR-corrected two-sided binomial p-value according to the C-test across the NFE and AFR subpopulations (out of N=15,865 genes), in intervals of deterministic $\hat{s}_{\text {het }}$ values derived from the NFE subpopulation of the ExAC dataset. FDR was controlled at 0.05 . 


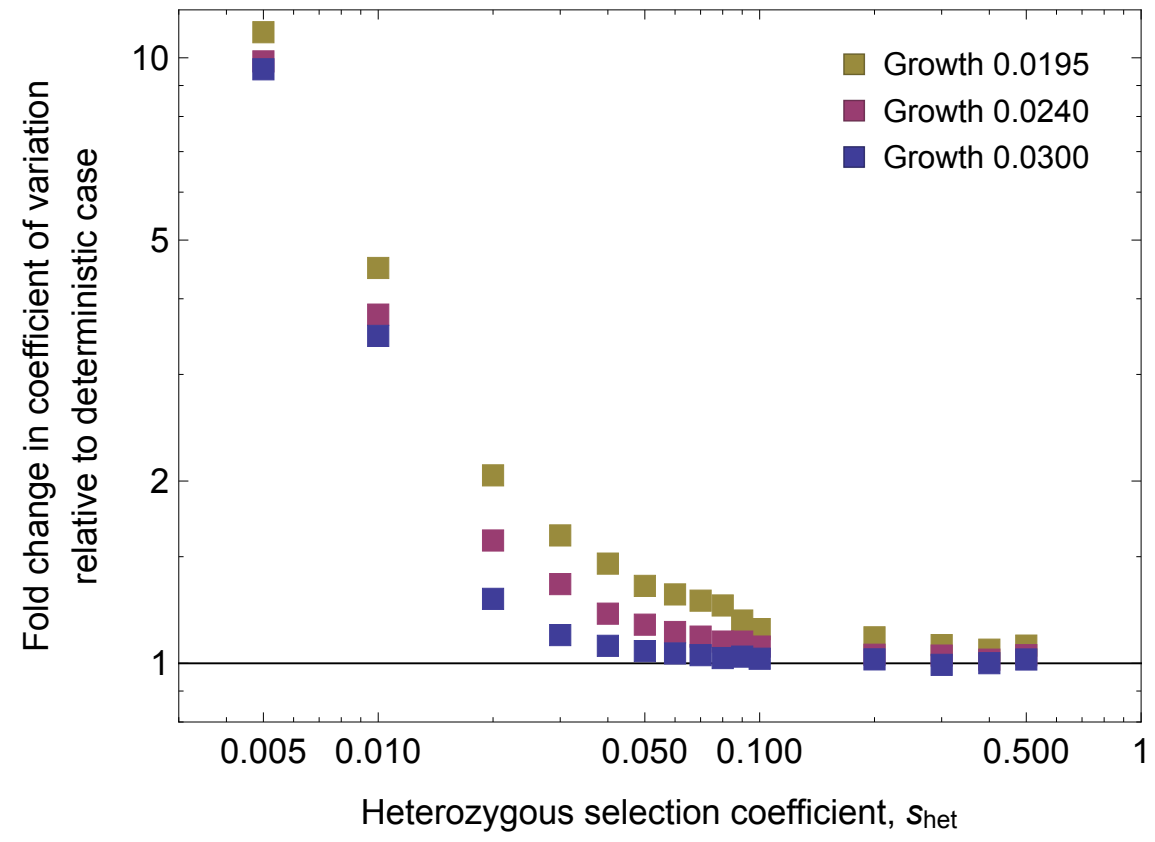

Supplementary Figure 1: Fold change in the coefficient of variation compared to the expectation under mutation-selection balance for different NFE demographies. Results are shown for three European demographies that differ in the rate of exponential expansion in the most recent exponential growth epoch. Inclusion of the effects of drift suggests a deviation from the Poisson assumption $\left(\operatorname{Var}[k] /\left(n U / s_{\text {het }}\right)>1.5\right)$ at approximately $s_{\text {het }}=0.02,0.03$, and 0.06 for growth rates of 0.030, 0.024, and 0.0195, respectively. Simulated points are shown (in order from left to right) for $s_{\text {het }}=\{0.005,0.01,0.02,0.03,0.04,0.05,0.06,0.07,0.08,0.09,0.1,0.2,0.3,0.4,0.5\}$. 


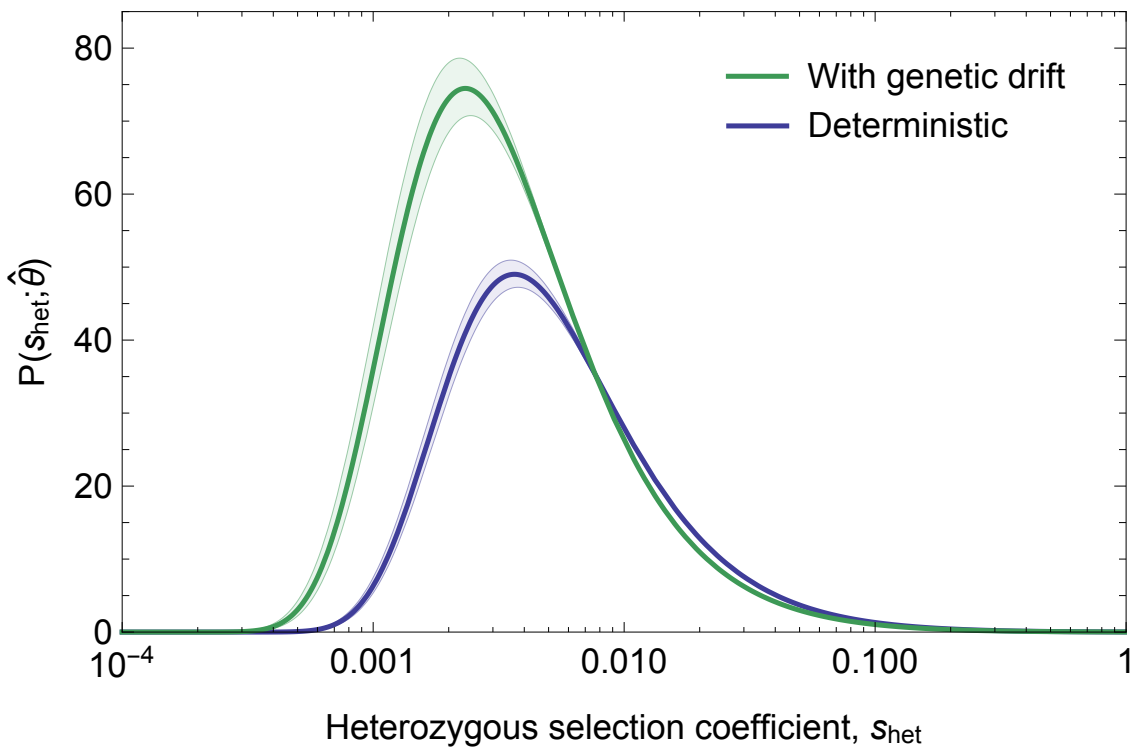

Supplementary Figure 2: Comparison between $P\left(s_{\text {het }} ; \hat{\boldsymbol{\theta}}\right)$ inferred under the mutation-selection balance assumption of Eq. 9 (blue) and when incorporating the effects of genetic drift, Eq. 8 (green). The latter scenario is based on a demographic model that fits the NFE subpopulation of the ExAC dataset and is in approximate correspondence with Harpak et al. (2016). Distributions are shown for fits to the PTV counts in the NFE sample, containing 16,279 genes with cumulative PTV allele frequency $\hat{X}<0.001$. Estimated parameters are $\hat{\boldsymbol{\theta}} \equiv(\hat{\alpha}, \hat{\beta})$, where $\alpha$ denotes the mean and $\beta$ the shape parameter of the inverse Gaussian distribution (Eq. 10). For the deterministic scenario we find $\left(\hat{\alpha}_{\operatorname{det}}=0.0699 \pm 0.0050, \hat{\beta}_{\mathrm{det}}=0.0105 \pm 0.0004\right)$, while including drift delivers $\left(\hat{\alpha}_{\text {drift }}=0.0573 \pm 0.0040, \hat{\beta}_{\text {drift }}=0.0070 \pm 0.0004\right)$, implying that the variance decreases by about $18 \%$ when stochasticity is accounted for. Parameter errors denote two s.d. from 100 bootstrapping replicates of the set of 16,279 genes, intended to quantify the influence of sampling noise in the data set on parameter inference, with fixed estimates of local mutation rate. Shaded areas in the plot show the corresponding confidence intervals. Note the logarithmic x-axis. 


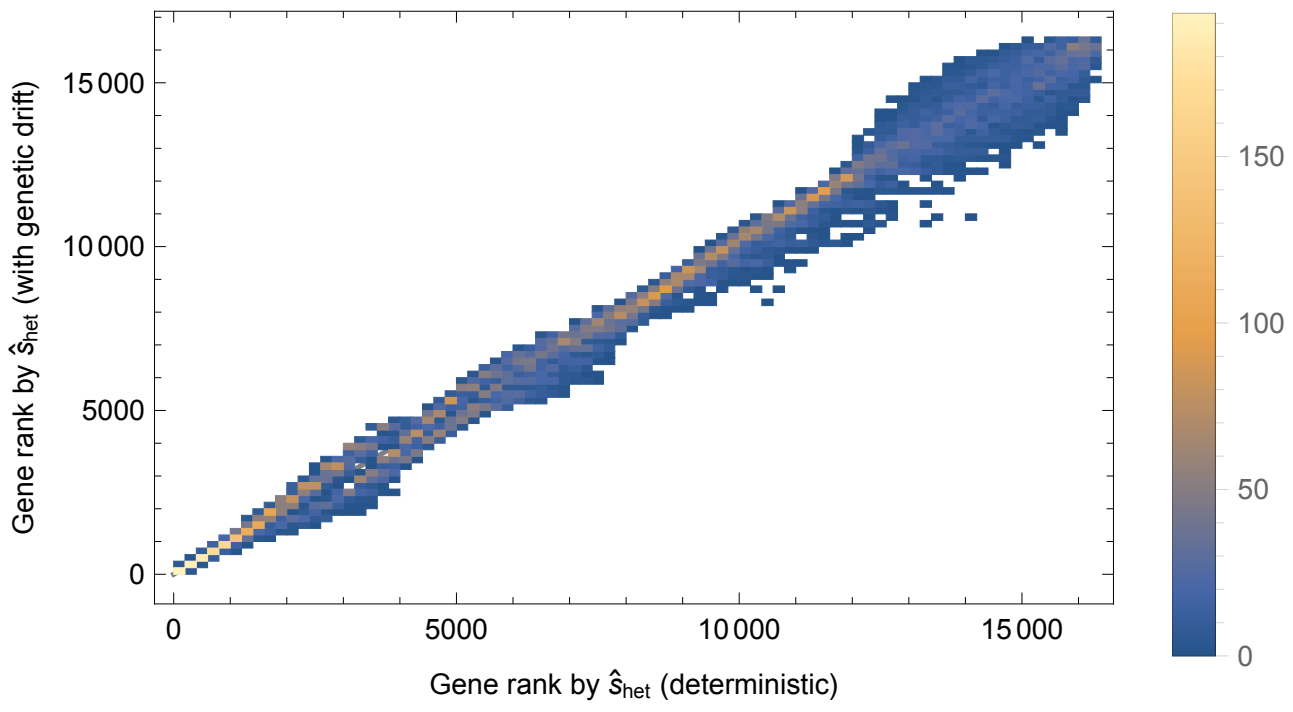

Supplementary Figure 3: Comparison between the ranks of per-gene selection estimates $\hat{s}_{\text {het }}$ for the NFE sample under the deterministic mutation-selection balance assumption (x-axis) and when incorporating the effects of genetic drift (y-axis). Shown is the density histogram from 16,279 genes. Spearman rank correlation between the estimates is 0.995 . 


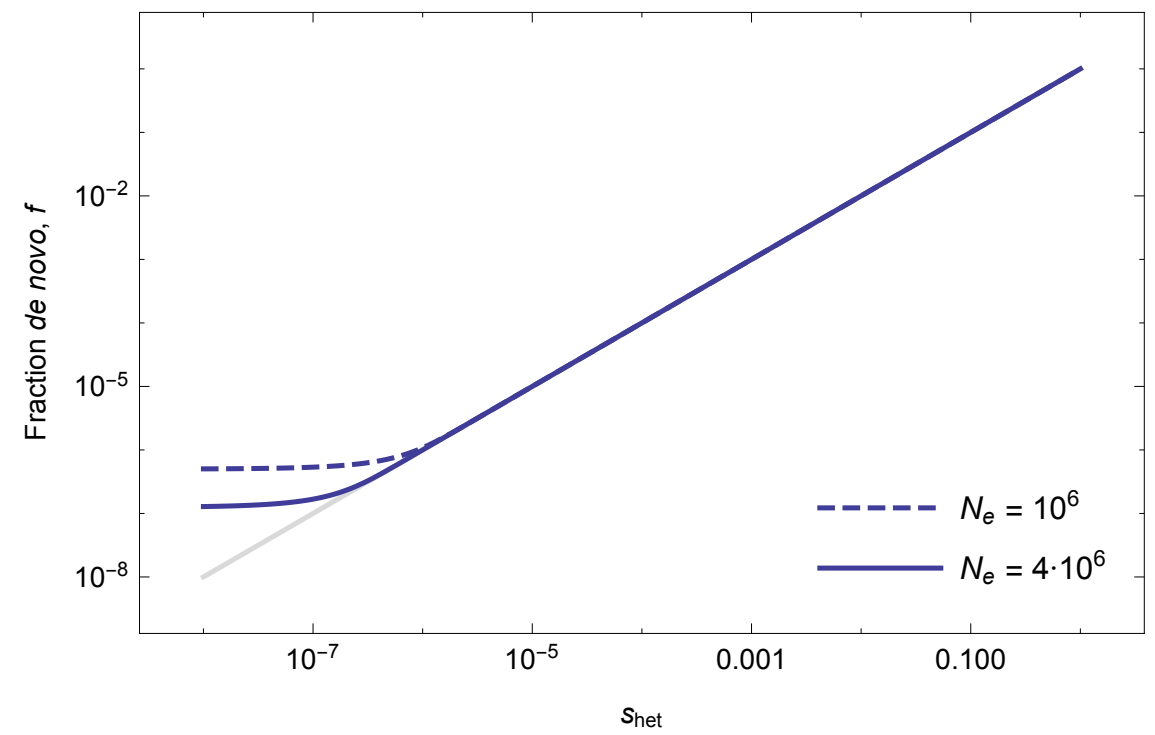

Supplementary Figure 4: Analytical expression for the fraction of de novo mutations, $f$, occurring under heterozygous negative selection strength $s_{\text {het }}$ in mutation-selection-drift balance for two different effective population sizes (solid blue line: $N_{\mathrm{e}}=4 \cdot 10^{6}$, dashed blue line: $N_{\mathrm{e}}=10^{6}$ ). The grey line shows the diagonal. For very weak selection, $f$ converges to the neutral equilibrium value of $1 /\left(1+2 N_{\mathrm{e}}\right)$. 


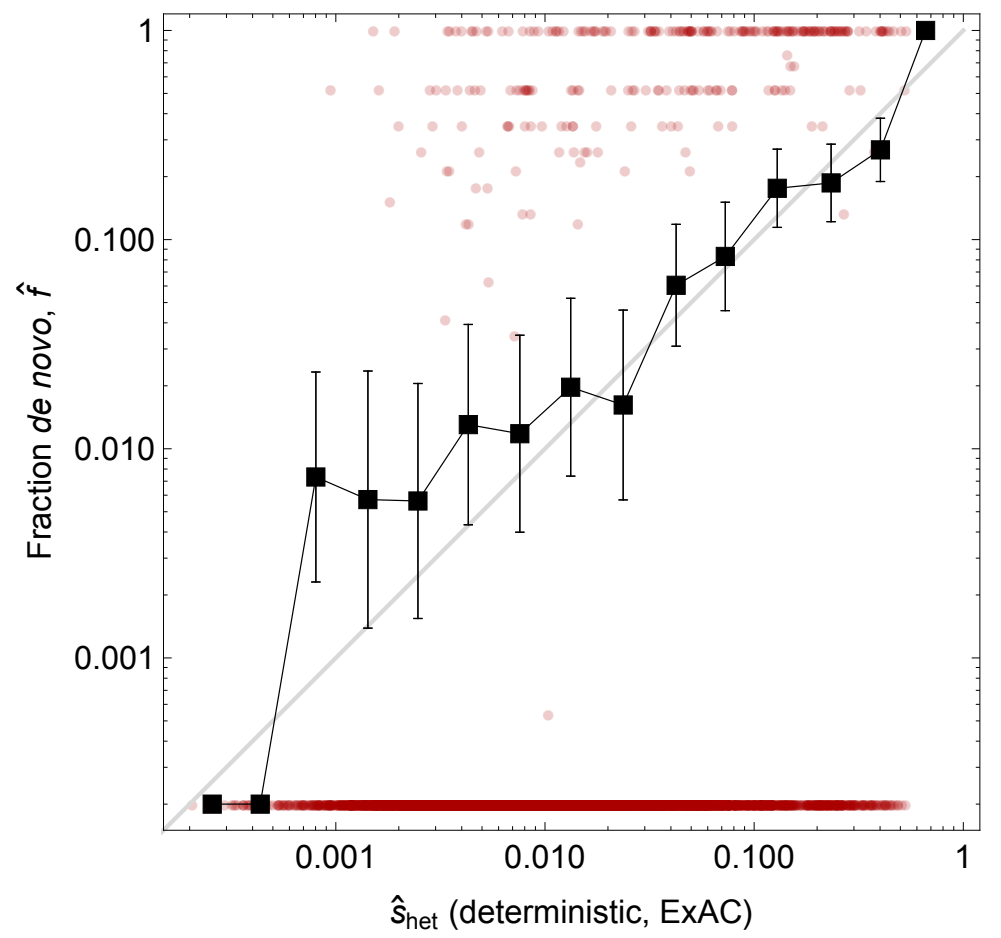

Supplementary Figure 5: De novo fraction $\hat{f}$ of PTV mutations was computed for 6,000 (out of 15,998) genes with at least one PTV (de novo or transmitted) in an autism-spectrum disorder cohort of about 4,000 parent-child trios (y-axis) and compared to the deterministic $\hat{s}_{\text {het }}$ derived from the full ExAC sample (Cassa et al., 2017) (x-axis). Red dots denote individual genes (genes with $\hat{f}=0$ were assigned $\hat{s}_{\text {het }}=2 \times 10^{-4}$ for illustration purposes). Black squares connected by black lines denote the mean in bins along the x-axis of logarithmic width $\Delta \log \left[\hat{s}_{\text {het }}\right]=0.25$ (number of genes per bin from left to right: $\{4,27,71,266,562,1023,1172,978,706,483,293,189,168$, $57,1\})$. Vertical error bars show the standard error of the mean per bin for $\hat{f}$. Corresponding error bars for $\hat{s}_{\text {het }}$ are smaller than the marker size. Grey line denotes the diagonal. 
(a)
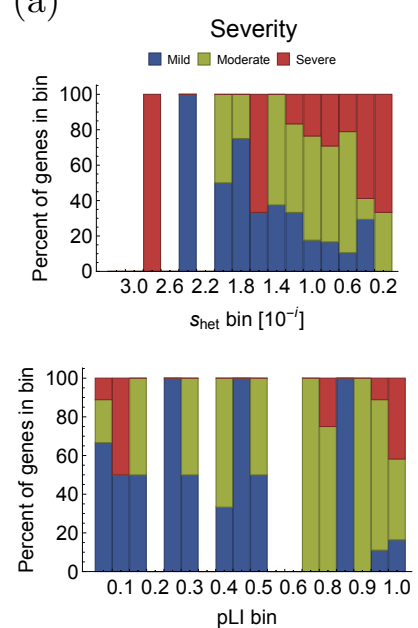

(b)
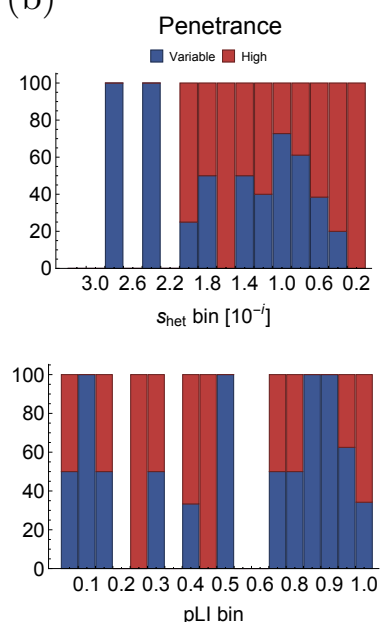

(c)
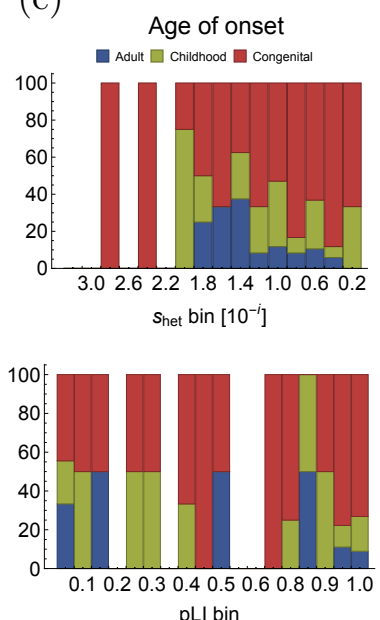

(d)
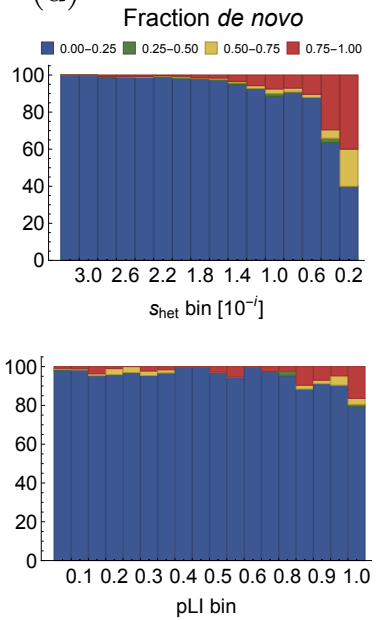

Supplementary Figure 6: Comparison of per-gene selection estimates, $\hat{s}_{\text {het }}$, with a measure of probability of loss-of-function intolerance, pLI (Lek et al., 2016). Shown is a different depiction of the respective correlation of NFE $\hat{s}_{\text {het }}$ and pLI with independent measures of gene importance from Figure 3. Genes are binned by their $\hat{s}_{\text {het }}$ values (top row, logarithmic bins of width 0.2 ) or pLI values (bottom row, linear bins of width 0.06) (x-axes). The y-axis shows the relative enrichment of each bin with the different categories of gene importance from each measure. (a-c) Data on disease severity, penetrance, and age of onset for a set of haploinsufficient disease-associated genes of high confidence (ClinGen Dosage Sensitivity Project). (d) The fraction of de novo PTVs, categorized by quartiles, was derived from an ASD trio-sequencing dataset. The gene set used for severity, penetrance and age of onset is enriched for high $\hat{s}_{\text {het }}$, reflected by the scarcity of observations in the low- $\hat{s}_{\text {het }}$ regime $\left(\hat{s}_{\text {het }}<10^{-2}\right)$ (a-c, top row). Most pLI values are located in the first and last bin $(\mathrm{pLI}<0.06$ and $\mathrm{pLI}>0.94)$, causing depletion in the intermediate pLI regime. Note the logarithmic $\mathrm{x}$-axis in the top row, while the bottom row has a linear $\mathrm{x}$-axis. 